\title{
Gerinnungs-Selbstmanagement \\ „Patienten machen das besser, als der Arzt es könnte“
}

Nach den neuen ESC-Leitlinien sind noch mehr Vorhofflimmernpatienten als bisher Kandidaten für Marcumar. Idealerweise sollten sie ihre INRWerte auch selbst kontrollieren, fordert Dr. J. H. Wirtz, stellvertretender Bundesvorsitzender des BNK.

MMW: Es wird oft kritisiert, dass Vorhofflimmernpatienten zu selten antikoaguliert werden. Ist der Vorwurf berechtigt? Wirtz: Ja, das ist er. Nur etwa $30 \%$ der Vorhofflimmernpatienten erhalten einen Vitamin-K-Antagonisten. Bei leitliniengemäßer Therapie müssten es aber mindestens doppelt so viele sein. Wir müssen mehr Patienten antikoagulieren. Das zeigt auch der Blick auf den neuen $\mathrm{CHA}_{2} \mathrm{DS}_{2}$-Vasc-Score in den ESC-Leitlinien zum Vorhofflimmern. Im Vergleich zum alten $\mathrm{CHADS}_{2}$-Score ist die Indikation für eine orale Antikoagulation noch erweitert worden. Frauen haben jetzt per se schon einen Punkt, für ein Alter über 65 gibt es ebenfalls einen Punkt, über 75 Jahre bereits zwei Punkte.
MMW: Warum erhalten so viele Patienten nur ASS statt Marcumar?

Wirtz: Viele Ärzte suchen eher nach Gründen, nicht antikoagulieren zu müssen, als nach Gründen für Marcumar. Ein Hauptargument gegen die Antikoagulation ist die Angst vor Blutungen: „Das ist doch eine alte Dame und die ist sturzgefährdet." Das aufwendige Monitoring und die damit für den Patienten verbundene Mühe ist ein weiterer wichtiger Grund.

MMW: In den neuen VorhofflimmernLeitlinien wird erstmals auch das Gerinnungs-Selbstmanagement als Option genannt. Was lässt sich damit erreichen? Wirtz: Es ist erwiesen, dass der Patient, der sich selbst managt, das viel besser macht, als jeder Arzt es machen könnte. Die Patienten erreichen dadurch häufiger ihren individuellen INR-Zielbereich. Das bedeutet, dass sie ein geringeres Risiko sowohl für Blutungskomplikationen als auch für Thrombosen haben. Ich bin ein großer Fan vom Gerinnungs-Selbstmanagement. Wir schulen bei uns sehr viele Patienten.

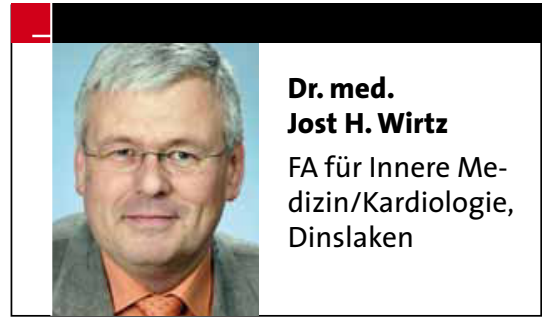

MMW: Eignet sich das Selbstmanagement denn auch für alte Patienten?

Wirtz: Man kann auch alte Menschen optimal schulen. Meine Schulungsassistentinnen haben mal untersucht, was die Schulungsteilnehmer nach zwei bis drei Jahren noch wissen. Interessanterweise wussten die Älteren und Alten noch viel mehr und befolgten das Gelernte auch viel penibler. Die Jungen waren nachlässiger und haben mehr Flüchtigkeitsfehler gemacht. Und wenn ein alter Mensch wirklich nicht geschult werden kann, z. B. ein 85-Jähriger mit fortgeschrittenem Parkinson, dann kann man zum Beispiel auch den Lebenspartner schulen.

- Interview: Dr. Beate Schumacher

\section{4-Stunden-Betamimetikum mit schnellem Wirkeintritt Mehr Luft für COPD-Patienten}

- Bronchodilatatoren bilden die Basis der medikamentösen COPD-Therapie. Seit einem Jahr steht der erste über 24 Stunden wirksame Beta-2-Agonist (LABA 24) Indacaterol (Onbrez ${ }^{\circledR}$ Breezhaler $^{\circledR}$ ) zur Verfügung. Bei einmal täglicher Anwendung erweitert er die Bronchien rasch und anhaltend, lindert die Luftnot und verbessert die Lungenfunktion. Die Substanz hat ihre Wirksamkeit im Vergleich zu Placebo und zu aktiven Vergleichssubstanzen in einem umfangreichen Studienprogramm mit insgesamt über 4000 Patienten gezeigt, wie Prof. C. Vogelmeier, Marburg, berichtete.

So konnte in der Phase-III-Studie INLIGHT-2 mit über 1000 Patienten belegt werden, dass mit der einmal täglichen
Gabe von $150 \mu \mathrm{g}$ Indacaterol nach zwölf Wochen eine signifikant größere Verbesserung der Lungenfunktion eingetreten war als bei zweimal täglicher Gabe von $50 \mu \mathrm{g}$ Salmeterol oder Placebo (beide $\mathrm{p}<$ o,001). Der Trough-FEV 1 war mit Indacaterol um $170 \mathrm{ml}$ größer als mit Placebo und um $60 \mathrm{ml}$ größer als mit Salmeterol (Kornmann O et al., Eur Respir J 2010).

Ebenso kam es mit dem LABA24 bei mehr Patienten zu einer klinisch relevanten Verbesserung der Lebensqualität um mindestens 4 Punkte im St. George's Respiratory Questionnaire, so Vogelmeier.

\section{Verringerung der Atemnot}

Ähnlich positive Ergebnisse zeigten sich auch in der zwölfwöchigen doppelblin- den Studie INSIST mit 1123 Patienten im COPD-Stadium II und III nach GOLD. Indacaterol einmal täglich erzielte eine bessere 24-Stunden-Bronchodilatation als Salmeterol zweimal täglich (Korn S et al., Poster ERS Barcelona, 2010). Der Anteil der Patienten mit einer klinisch relevanten Verringerung der Atemnot (Wertedifferenz von mindestens 1 auf dem Transition-Dyspnoe-Index) lag mit Indacaterol bei $69,4 \%$ im Vergleich zu $62,7 \%$ in der Salmeterolgruppe. Die Indacaterolpatienten erlebten zudem mehr Tage ohne Notfallmedikation $(p<0,05)$.

- Dagmar Jäger-Becker Quelle: Satellitensymposium, ERS-Kongress, Barcelona, 20. September 2010 (Veranstalter: Novartis) 